\title{
Toll-Like Receptor Signal Adaptor Protein MyD88 Is Required for Sustained Endotoxin-Induced Acute Hypoferremic Response in Mice
}

\author{
Antonio Layoun, ${ }^{* \dagger}$ Hua Huang, ${ }^{* \dagger}$ Annie Calvé, ${ }^{+\dagger}$ \\ and Manuela M. Santos ${ }^{\star \dagger \ddagger}$ \\ From the Montreal Cancer Institute, the Centre de recherche du \\ Centre hospitalier de l'Université de Montréal (CHUM) Research \\ Centre, ${ }^{\dagger}$ and the Department of Medicine, ${ }^{\neq}$University of \\ Montreal, Montreal, Quebec, Canada
}

Hypoferremia, associated with immune system activation, involves a marked reduction in the levels of circulating iron, coupled with iron sequestration within macrophages. Toll-like receptor (TLR) signaling plays an important role in the development of the hypoferremic response, but how downstream signaling events affect genes involved in iron metabolism is incompletely understood. We investigated the involvement of MyD88-dependent (MyD88) and MyD88independent (TRIF) TLR signaling in the development of hypoferremia. Using MyD88-deficient and TRIF-deficient mice, we show that MyD88 and TRIF signaling pathways are critical for up-regulation by lipopolysaccharide (LPS) of the iron regulator hepcidin. In addition, MyD88 signaling is required for the induction of lipocalin 2 secretion and iron sequestration in the spleen. Activation of TLR4 and TLR3 signaling through LPS and polyinosinic:polycytidylic acid [poly(I:C)] treatments resulted in rapid down-regulation of HFE protein [encoded by the hemochromatosis gene $(\mathrm{Hfe})]$ and ferroportin [encoded by solute carrier family $\mathbf{4 0}$ (iron-regulated transporter), member 1 (Slc40a1)] expression in the spleen, independent of MyD88 or TRIF signaling and proinflammatory cytokine production. However, lack of MyD88 signaling significantly impaired the hypoferremic response triggered by LPS, indicating that ferroportin and HFE protein down-regulation alone are insufficient to maintain hypoferremia. The extent of the hypoferremic response was found to be limited by initial, basal iron levels. Together, these results suggest that targeting specific TLR signaling pathways by affecting the function of adaptor molecules may provide new strategies to counteract iron sequestration within mac- rophages during inflammation. (Am J Pathol 2012, 180: 2340-2350; bttp://dx.doi.org/10.1016/j.ajpath.2012.01.046)

Inflammation is viewed as a protective response by the body necessary to remove, control, or neutralize injurious stimuli. ${ }^{1}$ Examples of harmful stimuli that can trigger an acute inflammatory response include invading pathogens, damaged cells, and irritants. Acute inflammation is triggered by the innate immune system, which represents the first line of defense in response to invading pathogens or tissue damage and plays a major role in host defense. $^{2}$ The innate immune response is activated through germline-encoded receptors, called pattern recognition receptors (PRRs). The PRRs recognize molecular patterns conserved throughout evolution in a wide range of microorganisms; these patterns are collectively termed pathogen-associated molecular patterns (PAMPs). Among the best characterized PRR families are the Toll-like receptors (TLRs), which are responsible for sensing invading pathogens both outside the cell and in intracellular endosomes and lysosomes. ${ }^{2}$ Each TLR recognizes specific PAMPs. For example, together with myeloid differentiation factor 2 (MD2), TLR4 recognizes extracellular lipopolysaccharide (LPS), which is a component of the outer membrane of Gram-negative bacteria. In addition, TLR4 is also involved in the recognition of viruses by binding to viral envelope proteins. TLR3 detects viral doublestranded RNA (dsRNA) in the endolysosome and is involved in the recognition of polyinosinic:polycytidylic acid [poly(l:C)], a synthetic dsRNA analog.

Supported by grants from the Canadian Institutes of Health Research (ClHR grant no. MOP44045) and the Natural Sciences and Engineering Research Council of Canada (NSERC grant no. 298515-2011 to M.M.S.) A.L. and H.H. are the recipients of Ph.D. scholarships from the NSERC. M.M.S. is the recipient of a Research Scholarship (Senior) from the FRSQ-Fonds de la recherche en santé du Québec.

Accepted for publication January 30, 2012.

Address reprint requests to Manuela Santos, Ph.D., Centre de recherche, CHUM-Hôpital Notre-Dame, Pav. De Sève Y5625, 1560 rue Sherbrooke est, Montréal H2L 4M1, QC, Canada. E-mail: manuela.santos@ umontreal.ca. 
After ligand binding, TLRs dimerize and undergo a conformational change required for the recruitment of downstream signaling molecules, which include a variety of adaptor molecules. Two major pathways have been identified, based on the usage of the distinct adaptor molecules MyD88 and TIR domain-containing adaptor inducing IFN- $\beta$ (TRIF; alias TICAM- 1 ). ${ }^{2}$ The MyD88 adaptor protein is essential for the downstream signaling of most TLRs. One exception is TLR3, which recruits TRIF adaptor protein in response to stimulation with dsRNA. The end result of these signaling cascades is the stimulation of gene expression and subsequent activation of antimicrobial and inflammatory activities.

Several of the genes that modify their expression levels during the TLR-mediated innate immune response are intrinsically linked to the control of iron metabolism. They are involved in the regulation of cellular iron uptake, release, and storage and play a pivotal role in the hypoferremic response. ${ }^{3}$ The hypoferremic response involves a marked reduction in the levels of extracellular iron associated with circulating iron levels and has been regarded as an important defense mechanism by which the host limits iron acquisition by invading pathogens. Iron is sequestered within macrophages during the hypoferremic response, resulting in limited iron availability for erythropoiesis. ${ }^{3}$ Although a transient hypoferremic response may be viewed as beneficial, long-term iron sequestration within macrophages becomes a critical factor contributing to the development of anemia of chronic disease or anemia of inflammation. ${ }^{4}$ Anemia of chronic disease is frequently found in patients with infections, malignancies, and autoimmune disorders and can substantially contribute to the morbidity of the underlying disease. In many instances, anemia of chronic disease is a predictor of adverse outcomes.

Mechanistically, iron diversion from the circulation into storage has been attributed to the production of proinflammatory cytokines such as TNF- $\alpha$ and IL-6 after immune activation. ${ }^{3}$ These proinflammatory cytokines regulate the levels of several iron metabolism genes, including the transferrin receptor (an iron importer), ${ }^{5,6}$ ferroportin (an iron exporter), ${ }^{7-9}$ and ferritin (an iron storage protein). ${ }^{10}$ Ferroportin binds to hepcidin, an antimicrobial peptide ${ }^{11,12}$ that induces ferroportin endocytosis and degradation. ${ }^{13}$ Hepcidin thus plays a pivotal role in controlling iron efflux from macrophages during the hypoferremic response.

In previous studies, we found that mice deficient in TLR4 are unable to regulate hepatic hepcidin expression in response to LPS, indicating that hepcidin induction in the liver is dependent on the signaling pathways mediated by TLR4.$^{14}$ TLR4-deficient mice retained the ability to mount a hypoferremic response, however, which suggests that acute changes in iron homeostasis elicited by LPS may occur through both TRL4-dependent and TRL4independent mechanisms. ${ }^{15}$ These studies led us to ask whether genes involved in iron metabolism may be regulated via divergent adaptor proteins and downstream signaling pathways triggered by TLR ligands.

In the present study, to gain further insight into the mechanisms involved in the acute hypoferremic re- sponse, we investigated the role of the alternative TLR adaptors MyD88 and TRIF, by using MyD88 $8^{-\prime}$ and TRIFdeficient mice. In addition, HFE protein and lipocalin-2 (Lcn2), two important proteins in iron metabolism ${ }^{16,17}$ and iron sequestration during infection, ${ }^{18,19}$ were also examined for their potential role during the acute hypoferremic response using $\mathrm{Hfe}^{-/-}$and $\mathrm{LCn}^{-/-}$mice.

\section{Materials and Methods}

\section{Animals}

All procedures were performed in accordance with the Canadian Council on Animal Care guidelines after approval by the institutional Animal Care Committee of the CHUM Research Center. C57BL/6 (B6) WT (WT) female mice aged between 6 and 8 weeks were purchased from Charles River Laboratories (Wilmington, MA). TRIF-deficient mice (C57BL/6J-Ticam1Lps2/J) were purchased from Jackson Laboratories (Bar Harbor, ME). MyD88 ${ }^{-/-}$ mice and $L c n 2^{-/-}$mice with the B6 genetic background were kindly provided by Dr. Shizuo Akira (Research Institute for Microbial Diseases, Osaka University, and Japan Science and Technology Agency, Tokyo, Japan) and were maintained as described previously. ${ }^{18,20} \mathrm{Hfe}^{-/-}$ mice in the 129/SvEvTac background were kindly provided by Dr. Nancy C. Andrews (Howard Hughes Medical Institute and Harvard Medical School, Children's Hospital, Boston, MA $)^{21}$ and were backcrossed onto the B6 background for 10 generations at our animal facility. ${ }^{14} \mathrm{All}$ animals used in the experiments were female and were permanently housed under specific pathogen-free conditions.

\section{Animal Treatments}

Mice were fed a commercial diet (Teklad Global 18\% protein rodent diet; Harlan Teklad, Madison, WI). Where indicated, dietary iron overload was produced by giving 8-week-old mice the same commercial diet supplemented with $2.5 \%(\mathrm{w} / \mathrm{w})$ carbonyl iron (Sigma-Aldrich, St. Louis, MO) for 2 weeks. ${ }^{22}$

Acute inflammation was produced by a single intraperitoneal injection of different TLR ligands: LPS, poly(I:C), or zymosan. The LPS (Escherichia coli serotype 055:B5; 1 $\mu \mathrm{g} / \mathrm{g}$ unless otherwise indicated) was purchased from Sigma-Aldrich); Sterile poly(l:C), $12.5 \mu \mathrm{g} / \mathrm{g}$ (Endotoxin level: EndoFit $<0.001$ endotoxin units $(\mathrm{EU}) / \mu \mathrm{g}$ endotoxin); and zymosan (250 $\mu \mathrm{g} / \mathrm{g}$; Endotoxin level: EndoFit < $0.001 \mathrm{EU} / \mu \mathrm{g}$ endotoxin) were purchased from InvivoGen (San Diego, CA). In other experiments, mice were injected with recombinant mouse IL-6 (1 $\mu \mathrm{g} / \mathrm{g}$ i.p.; Cedarlane Laboratories, Burlington, ON, Canada). Control mice were injected with an equivalent volume of sterile saline solution $(0.9 \% \mathrm{NaCl})$.

\section{IL-6 Assay}

IL-6 protein levels in serum were measured using mouse IL-6 enzyme-linked immunosorbent assay kits according 
to the manufacturer's instructions (R\&D Systems, Minneapolis, MN).

\section{SDS-PAGE and Western Blot Analysis}

Protein concentrations were measured using a Pierce BCA protein assay kit (Thermo Fisher Scientific, Rockford, IL). Equivalent amounts of protein from tissue extracts were loaded to perform electrophoresis. For analysis of Lcn2 in serum, $1.5 \mu \mathrm{L}$ of serum was boiled in loading buffer containing 4\% SDS, 20\% glycerol, and bromophenol blue for 5 minutes. Proteins were resolved on $12 \%$ SDS-PAGE gels and then were transferred onto nitrocellulose membranes (GE Healthcare; Amersham Biosciences, Baie d'Urfé, QC, Canada). The membranes were blocked with $5 \%$ nonfat dry milk solution and incubated with antibodies against ferroportin (a kind gift from Dr. Kostas Pantopoulos, McGill University), ${ }^{23}$ HFE protein (Alpha Diagnostic, San Antonio, TX), Lcn2 (R\&D Systems), and $\beta$-actin (Abcam, Cambridge, MA). To detect immunocomplexes formed, peroxidase-conjugated anti-rabbit IgG (Cell Signaling Technology, Danvers, MA), anti-mouse IgG (GE Healthcare, Little Chalfont, UK) or anti-goat IgG (R\&D Systems) were used as secondary antibodies. Staining intensity was developed with an Amersham enhanced chemiluminescence system (GE Healthcare).

\section{Quantitative RT-PCR}

Total RNA from tissue samples was isolated by acid phenol extraction ${ }^{24}$ (TRIzol; Invitrogen, Burlington, ON, Canada) as recommended by the manufacturer, and reverse transcription was performed with an Omniscript RT-PCR system (Qiagen, Mississauga, ON, Canada). mRNA levels of selected genes were measured by realtime PCR with a Rotor Gene 3000 real-time DNA detection system (Montreal Biotech, Kirkland, QC, Canada) and QuantiTect SYBR Green I PCR kits (Qiagen), as described previously ${ }^{25}$ and using primer sequences listed in Table 1. Expression levels were normalized to the housekeeping gene $\beta$-actin (ACTB).

\section{Serum Iron and Total Iron Binding Capacity}

Serum iron and total iron-binding capacity were assessed by colorimetric assay with a Kodak Ektachem DT60 system (Johnson \& Johnson, Ortho Clinical Diagnostics, Mississauga, ON, Canada), and transferrin saturation was calculated from these data. ${ }^{22}$

\section{Measurement of Iron Concentration in the Spleen}

Splenic iron concentration was assessed by acid digestion of tissue samples, followed by iron quantification with atomic absorption spectroscopy. ${ }^{15}$

\section{Statistical Analysis}

All statistics were calculated using SigmaStat version 3.1 (Systat Software, Richmond, CA). Multiple comparisons were evaluated by one-way analysis of variance, followed by the Bonferroni multiple comparison test.

\section{Results}

\section{TLR Agonists Trigger Hypoferremic Responses in Mice}

To investigate the involvement of TLRs and associated signaling pathways in hypoferremia, we first studied hypoferremic responses to activation of TLR2 (MyD88 signaling), TLR3 (TRIF signaling), and TLR4 (MyD88 and TRIF signaling) by treating mice with zymosan (TLR2 agonist), poly(I:C) (TLR3 agonist), or LPS (TLR4 agonist). Serum iron levels and transferrin saturation were significantly lower in mice treated with TLR ligands, compared with saline-treated controls (Figure 1, A and B). Thus, stimulation with TLR ligands resulted in a significant reduction of circulating iron. These results indicate that both MyD88 and TRIF signaling can generate an acute hypoferremic response.

\section{MyD88 Signaling Is Required for Sustained LPS-Induced Hypoferremic Response}

To further understand the involvement of MyD88 and TRIF-mediated signaling in the hypoferremic response, we next examined the role of these two major pathways during LPS-induced acute hypoferremia using mice deficient in either MyD88 (MyD88 ${ }^{-/-}$) or TRIF (Trif ${ }^{L p s 2 / L p s 2}$ ) protein. Mice were treated with LPS or saline solution, and serum iron levels and transferrin saturation were measured at 1.5, 3, 6, 12, and 24 hours after injection (Figure 2, A and B). The hypoferremic response in Trif $^{L p s 2 / L p s 2}$ mice was very similar to that observed in WT mice, with a sharp drop in circulating iron that reached maximum reduction at 6 hours after treatment and persisted for up to 24 hours. However, although MyD88 ${ }^{-1-}$ mice responded with an initial drop of serum iron param-

Table 1. Genes and Primer Sequences Used in Quantitative Real-Time PCR

\begin{tabular}{lll}
\hline Gene (protein) & Forward & \multicolumn{1}{c}{ Reverse } \\
\hline Actb $(\beta$-actin) & $5^{\prime}$-TGTTACCAACTGGGACGACA-3' & $5^{\prime}$-GGTGTTGAAGGTCTCAAA-3' \\
Hamp (hepcidin) & $5^{\prime}$-AGAGCTGCAGCCTTTGCAC-3' & $5^{\prime}$-GAAGATGCAGATGGGGAAGT-3' \\
Slc40a1 (ferroportin) & $5^{\prime}$-CCCATCCCCATAGTCTCTGT-3' & $5^{\prime}$-CTTGCAGCAACTGTGTCACC-3' \\
Hfe (HFE protein) & $5^{\prime}$-TCTTGGATCTCCACGTTC-3' & $5^{\prime}$-TCATCCACATAGCCCCTAGC-3' \\
Lcn2 (lipocalin-2) & $5^{\prime}$-CCTCAAGGACGACAACATCA-3' & $5^{\prime}$-ACCCATTCAGTTGTCAATGC-3' \\
\hline
\end{tabular}




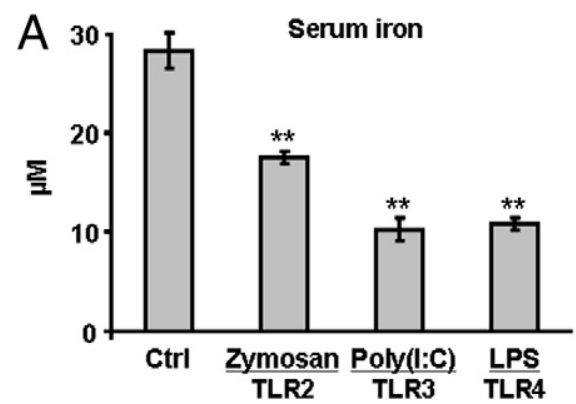

eters up to 3 hours after LPS injection, they were unable to sustain this response. In fact, serum iron levels in MyD88-deficient mice started rising again after 3 hours and even increased significantly above basal levels after 24 hours $(P<0.05)$. Thus, lack of MyD88 signaling significantly impairs the hypoferremic response triggered by LPS.

\section{Dual Signaling of MyD88 and TRIF Is Critical for Activation of the Iron Regulator Hepcidin during LPS-Induced Hypoferremia}

Having established that MyD88 is important for maintaining low circulating iron levels during the acute response to LPS, we investigated the mechanism associated with the suppressed hypoferremic response in $M y D 88^{-1-}$ mice. To this end, we assessed levels of hepcidin, the major regulatory hormone involved in iron metabolism. ${ }^{4}$ Hepcidin levels rose significantly in WT mice 3 and 6 hours after LPS treatment (Figure 2C), confirming previous work demonstrating the ability of LPS to induce hepcidin. ${ }^{14,26}$ Unexpectedly, both MyD88 ${ }^{-/-}$and Trif ${ }^{L p s} 2 / L p s 2$ mice failed to up-regulate their hepatic hepcidin mRNA levels, with hepcidin levels remaining constant for up to 24 hours.

To further confirm the need for both MyD88 and TRIF signaling to activate hepcidin, we measured hepatic hepcidin levels in WT and MyD88 ${ }^{-1-}$ mice stimulated with poly (I:C) to activate the TRIF pathway. Poly(I:C) caused a significant reduction in circulating iron, as judged by the reductions in serum iron and transferrin saturation seen at 6 hours after injection (Figure 3, A and B). In the liver and spleen, however, hepcidin mRNA levels remained unchanged in both mouse strains (Figure 3, C and D). Taken together, these results indicate that signaling through both adaptor molecules (ie, both MyD88 and TRIF) is necessary to achieve hepcidin induction, suggesting that there may be hepcidin-independent pathways participating in the acute hypoferremic response in mice.

\section{IL-6 Production Is Suppressed in MyD88 ${ }^{-1-}$

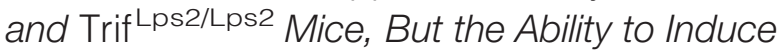 Hepcidin in Response to IL-6 Is Retained}

Given that hepcidin elevation during inflammatory conditions has been shown to be mediated mainly by IL- $6,{ }^{27}$ we examined whether IL-6 production stimulated by LPS in $M y D 88^{-1-}$ and Trif ${ }^{L s 2 / L p s 2}$ mice was compromised and could thus explain the lack of hepcidin activation in these mice. Serum IL-6 levels, which peaked at 6 hours after treatment in WT mice, were indeed severely impaired in both $M y D 88^{-/-}$and Trif ${ }^{L p s 2 / L p s 2}$ mice (Figure 4A), with the lowest levels being observed in $M y D 88^{-1-}$ mice. Next, to test whether $M y D 88^{-1-}$ and Trif ${ }^{L p s 2 / L p s 2}$ mice maintain the ability to elevate hepcidin in response to $\mathrm{IL}-6$, we treated mice with recombinant mouse IL-6. In all mouse strains, IL-6 treatment led to a mild but significant decrease in circulating iron, as well as to induction of hepcidin (Figure 4, B and C), indicating that $M y D 88^{-1-}$ and Trif ${ }^{L P S 2 / L p s 2}$ mice retain the ability to regulate hepcidin levels in response to IL-6.


Figure 2. MyD88-deficient mice are unable to sustain the acute hypoferremic response induced by LPS. Serum iron levels (A), transferrin saturation (B), and hepatic hepcidin mRNA levels $(\mathbf{C})$ were measured in WT, $M y D 88^{-/-}$, and Trif ${ }^{L p s} 2 / L s 2$ mice at $1.5,3,6,12$, and 24 hours after LPS or saline (Ctrl) injection. Data are expressed as means \pm SEM. Results are representative of three independent experiments. ${ }^{*} P<0.05,{ }^{* * *} P<0.001$ versus saline-treated mice $(\mathrm{Ctrl})$. ${ }^{\dagger} P<0.001$ $M y D 88^{-1-}$ versus WT or Trif ${ }^{L p s / L p s 2}$ mice. $n=4$ mice per group. 


\section{A Serum iron}

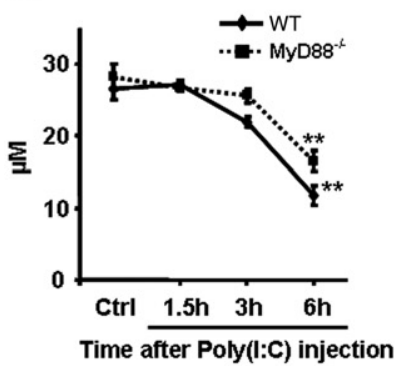

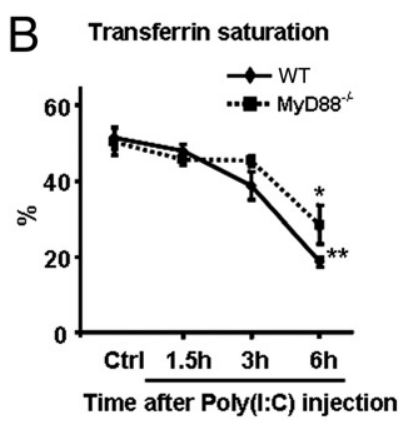

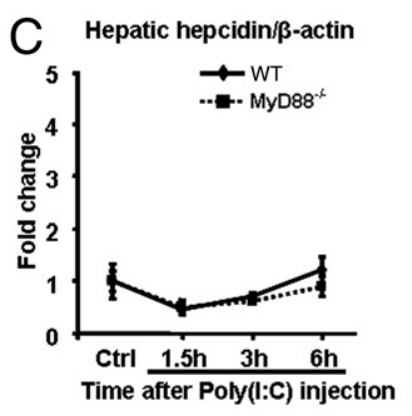

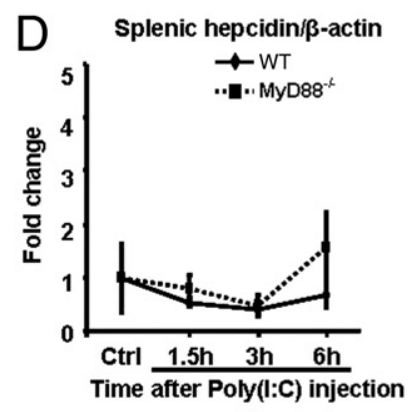

Figure 3. TLR3 activation induces acute hypoferremia in WT and $M y D 88^{-/-}$mice without hepcidin induction. Serum iron levels (A), transferrin saturation (B), and hepcidin mRNA levels in liver (C) and in spleen (D) of WT and $M y D 88^{-/}$mice were measured at 1.5, 3, and 6 hours after saline (Ctrl) or poly(I:C) treatment. Data are expressed as means \pm SEM. Results are representative of three similar experiments. ${ }^{*} P<0.05,{ }^{* *} P<0.001$ versus saline-treated mice (Ctrl). $n=4$ mice per group.

\section{Suppression of the Iron Exporter Ferroportin by LPS Is Independent of MyD88 and TRIF Signaling}

Ferroportin, which is most likely the only cellular iron exporter in mammals, ${ }^{28}$ also plays a major role during the hypoferremic response. ${ }^{7-9}$ Ferroportin mRNA levels have been reported to be down-regulated in macrophages during inflammation, thereby limiting iron efflux and increasing iron sequestration within macrophages. ${ }^{29,30} \mathrm{We}$ therefore measured ferroportin levels during the acute response to LPS in the spleen, a macrophage-rich organ that expresses high ferroportin levels. ${ }^{8}$ Both WT and $M y D 88^{-1-}$ mice exhibited a sharp reduction of mRNA ferroportin expression between 1.5 and 12 hours after LPS treatment (Figure 5A). Furthermore, ferroportin protein levels also decreased in the spleens of both WT and $M y D 88^{-1-}$ mice (Figure 5, B-D). To further investigate the requirement for TRIF signaling for ferroportin mRNA down-regulation, we elicited TRIF signaling through TLR3 by treating WT and Trif ${ }^{L p s 2 / L p s 2}$ mice with poly(I:C). Both mouse strains were able to significantly down-regulate ferroportin mRNA in the spleen at 6 hours after poly $(\mathrm{I}: \mathrm{C})$ injection (Figure 5E). Taken together, these results indicate that ferroportin down-regulation during acute hypoferremia is independent of MyD88 or TRIF signaling.

Next, we measured iron concentrations in the spleen, which represents a major site of iron storage during in- flammation. ${ }^{31}$ Iron levels rose significantly in WT and Trif ${ }^{L p s 2 / L p s 2}$ mice at 12 and 24 hours after LPS injection, albeit to a lesser extent in Trif ${ }^{L p s 2 / L p s 2}$ mice at 24 hours, compared with WT mice, but remained unchanged in $M y D 88^{-/-}$mice (Figure 5F).

The Hereditary Hemochromatosis Gene (Hfe) Is Down-Regulated during the Acute Hypoferremic Response without Requiring MyD88 and TRIF Signaling

HFE protein is involved in cellular iron uptake, at least in cell culture models, ${ }^{16,17}$ through regulation of transferrin receptor 1 (TfR1)-mediated uptake of transferrin-bound iron, ${ }^{32}$ as well as in iron release. ${ }^{33}$ Because hypoferremia is associated with removal of iron from circulation, we reasoned that HFE protein may participate in the development of hypoferremia by enhancing the ability of macrophages to take up iron via their interaction with TfR1. We therefore investigated whether HFE protein levels change in the spleens of mice treated with LPS. Hfe mRNA and HFE protein levels in the spleen were significantly down-regulated in both WT and MyD88 ${ }^{-1-}$ mice (Figure 6, A and B). Similarly, poly(I:C) treatment significantly reduced Hfe mRNA expression in the spleen of both WT and Trif ${ }^{L p s 2 / L p s 2}$ mice (Figure $6 C$ ). These results suggest that, similarly to ferroportin, HFE protein down-
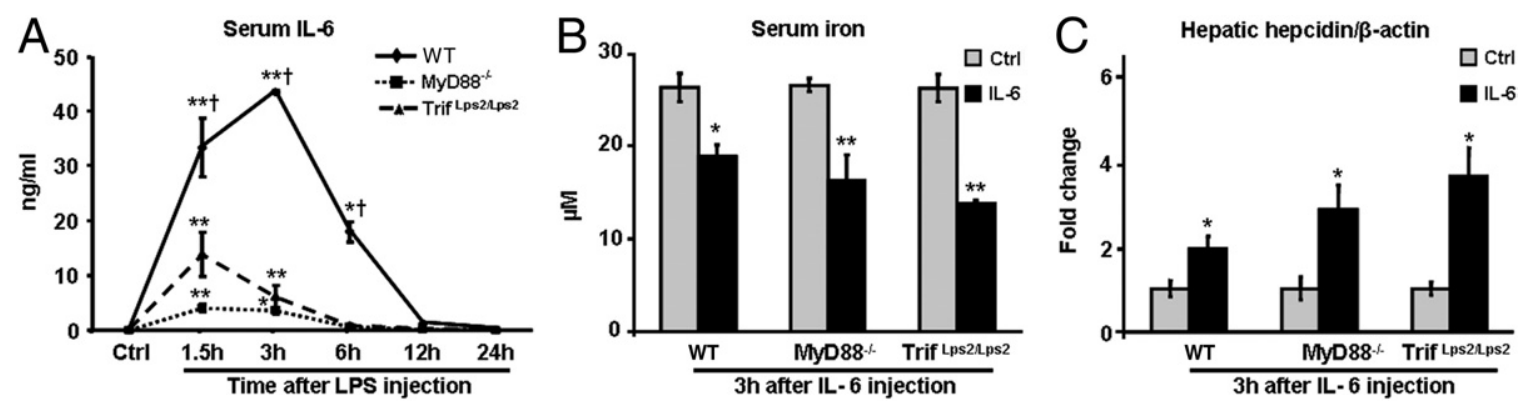

Figure 4. $M y D 88^{-/-}$and Trif Lps2/Lps 2 mice fail to produce IL-6 after LPS treatment, but retain the ability to respond to IL-6 stimulation. A: IL-6 serum levels in WT, $M y D 88^{--}$, and Trif ${ }^{L \text { Ls } 2 / L s 2}$ mice were measured at $1.5,3,6,12$, and 24 hours after LPS or saline (Ctrl) injection. Results are representative of three independent experiments. Serum iron levels (B) and hepcidin mRNA levels in the liver $(\mathbf{C})$ of WT, $M y D 88^{-1-}$, and Trif $f^{L p s} / L p s 2$ mice were measured at 3 hours after saline (Ctrl) or IL-6 treatment. Data are expressed as means \pm SEM. ${ }^{*} P<0.05,{ }^{* *} P<0.001$ versus saline-treated mice $(\mathrm{Ctrl}) ;{ }^{\dagger} P<0.05 \mathrm{WT}$ versus $M y D 88^{-/-}$ or Trif ${ }^{L p s / L p s 2}$ mice. $n=4$ mice per group. 

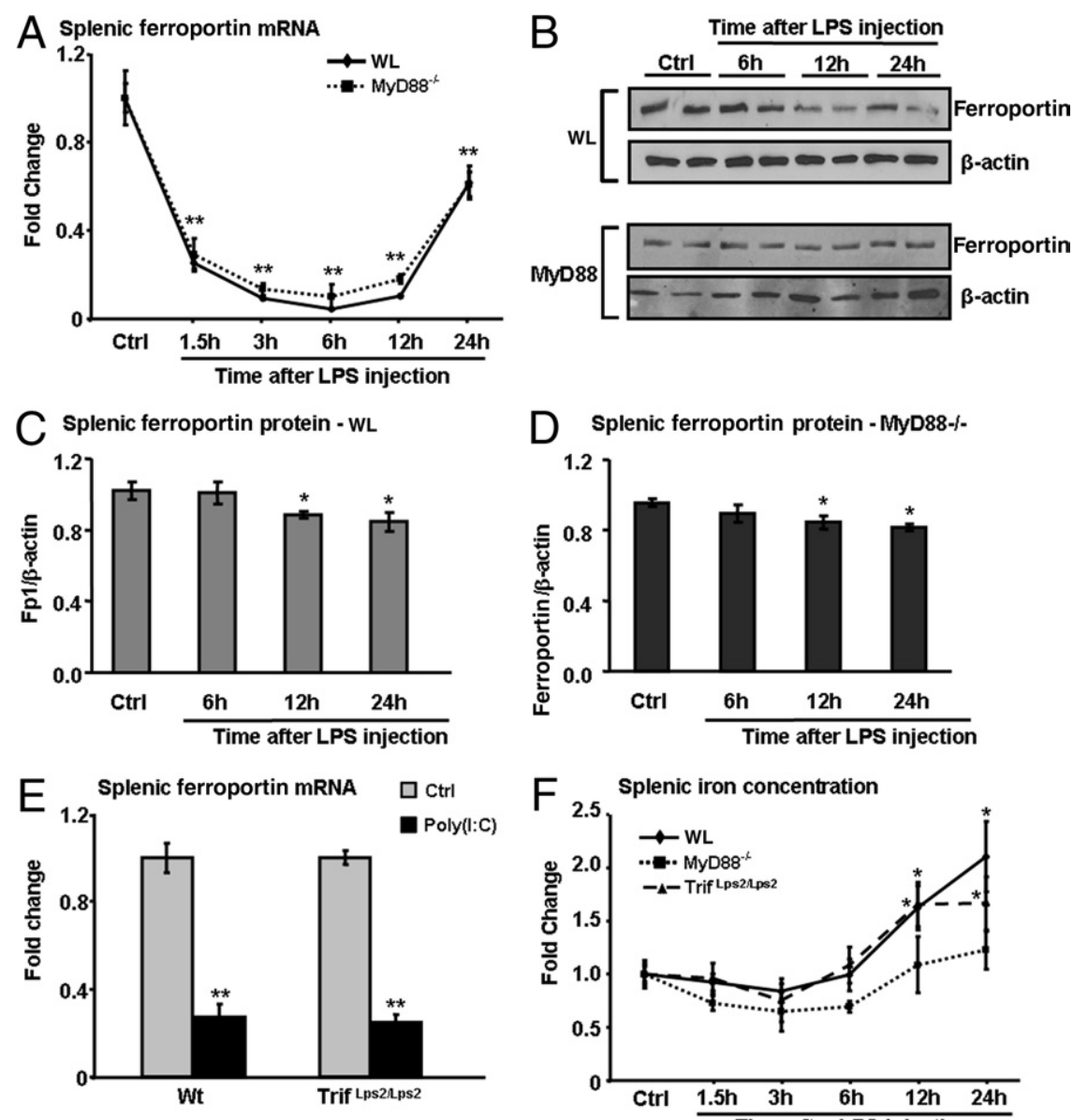

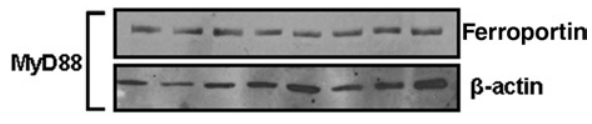

D Splenic ferroportin protein - MyD88-\%-
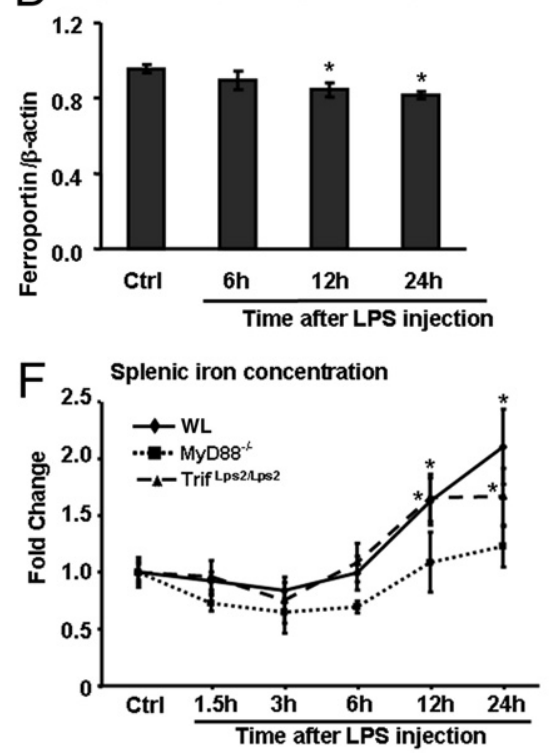

Figure 5. MyD88 and TRIF signaling are not required for ferroportin down-regulation, but MyD88 is critical for iron storage in the spleen. Ferroportin mRNA (A) and protein levels (B) in the spleen of WT and $M y D 88^{-/-}$mice treated with saline (Ctrl) or LPS. Ferroportin protein expression levels were calculated relative to $\beta$-actin in WT (C) and $M y D 88^{-/-}$(D) mice using Western blots from two independent experiments. E: Ferroportin mRNA levels in the spleen of WT and Trif ${ }^{L p s 2 / L p s 2}$ mice at 6 hours after treatment with saline (Ctrl) or poly(I:C). Data are expressed as means \pm SEM. Results are representative of three similar experiments. F: Splenic iron concentration in WT, Trif ${ }^{L \text { Ls } 2 / L p s 2}$, and $M y D 88^{-1-}$ mice was measured at $1.5,3,6,12$, and 24 hours after LPS or saline (Ctrl) injection. Data are expressed as means \pm SEM. Results are representative of three similar experiments. ${ }^{*} P<$ 0.05 , ${ }^{* * *} P<0.001$ versus saline-treated mice $(\mathrm{Ctrl})$ $n=4$ mice per group or time point. regulation during the acute hypoferremic response occurs independent of MyD88 and TRIF signaling pathways.

\section{The Reduction in Circulating Iron during the Hypoferremic Response Is Limited by Basal Iron Levels but Not by HFE Protein}

To further elucidate the potential role of HFE protein during hypoferremia, we used $\mathrm{Hfe}^{-1-}$ mice and followed their response to LPS treatment. $\mathrm{Hfe}^{-\prime-}$ mice retained the ability to lower their circulating iron after LPS treatment, albeit their serum iron values were consistently more elevated than those found in WT mice measured at the same time point (Figure 6D). Because HFE-deficient mice have higher basal levels of circulating iron than WT mice, we wondered whether the inability of $\mathrm{Hfe}^{-/-}$ mice to reduce serum iron to approach values seen in WT mice in response to LPS was due to their higher initial iron levels. To address this question, we placed WT mice on an iron-enriched diet for 2 weeks and then treated them with LPS. Similarly to what we had observed in $\mathrm{Hfe}^{-/-}$mice, iron-loaded WT mice were still able to significantly reduce serum iron levels; however, levels remained consistently above those observed in mice that were not iron loaded (Figure 6D). Importantly, when data were analyzed in terms of fold changes, the reduction of serum iron levels was indistinguishable among WT mice maintained on the standard diet, ironloaded WT mice, and $\mathrm{Hfe}^{-\prime-}$ mice (Figure 6E). These results suggest that the initial, basal serum iron level (rather than the absence of Hfe expression) is the principal factor determining the differences observed between WT and $\mathrm{Hfe}^{-/-}$mice.

\section{MyD88 Signaling Is Critical for the Induction of the Iron Siderophore Binding Protein Lcn2}

During bacterial infection, mammalian spleen and macrophages synthesize abundant amounts of Lcn2, an antimicrobial protein that has been shown to mediate an innate immune response to bacterial infection by sequestering circulating iron. ${ }^{18,19}$ To determine whether iron sequestration by Lcn2 may contribute to the rapid drop in serum iron observed during acute inflammation, we first measured splenic mRNA and serum protein levels of Lcn2 in LPS-treated WT and MyD88 ${ }^{-/-}$mice. Lcn2 production upon LPS stimulation was severely impaired in MyD88-deficient mice (Figure 7, $A$ and $B$ ), indicating that Lcn2 induction requires MyD88 signaling. 

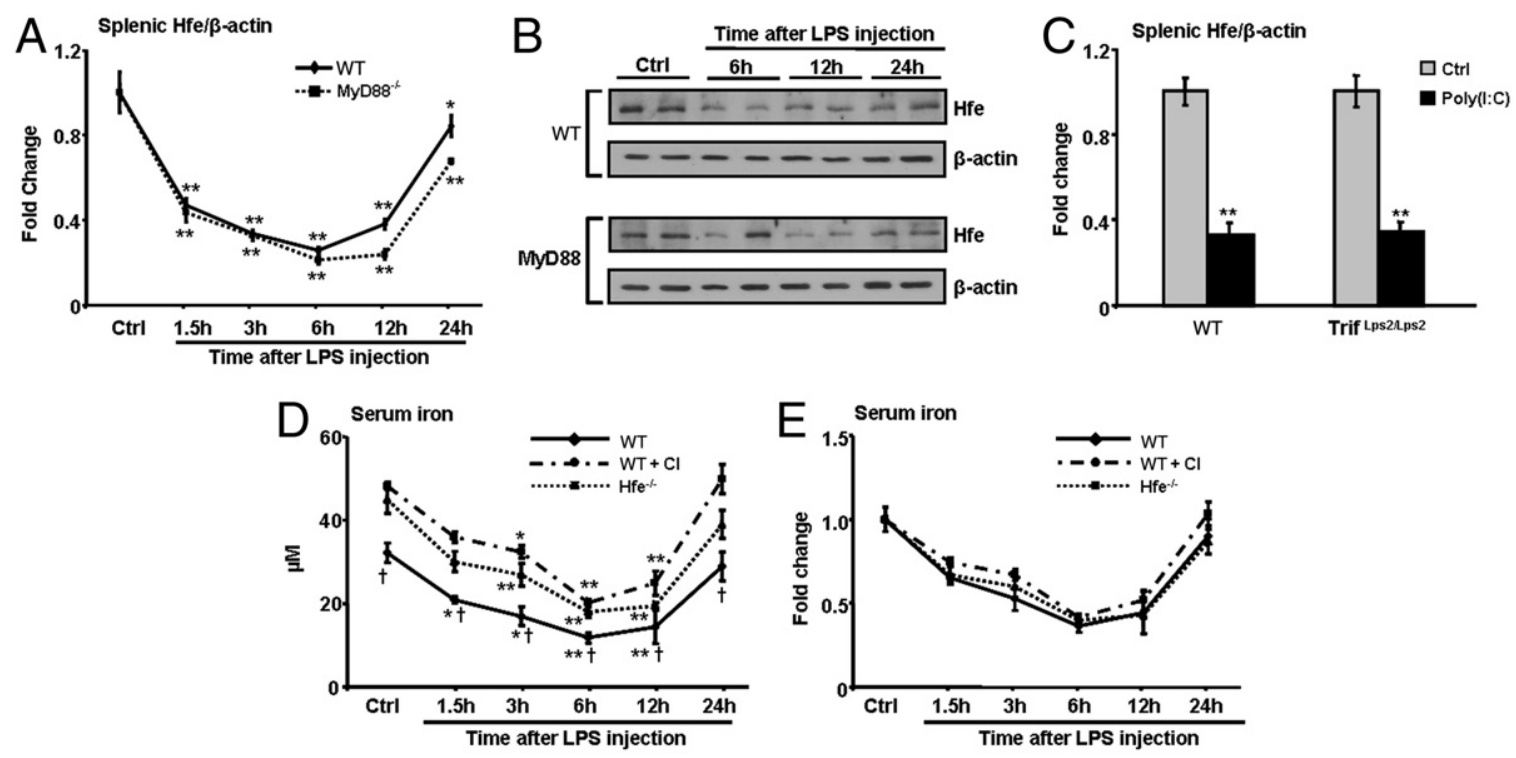

Figure 6. The role of HFE protein in the hypoferremic response. Hfe mRNA (A) and HFE protein levels (B) in the spleen of WT and $M y D 88^{-1-}$ mice treated with saline (Ctrl) or LPS. C: Hfe mRNA levels in the spleen of WT and Trif L ${ }^{L 2 / L p s 2}$ mice at 6 hours after poly(I:C) treatment. Data are expressed as means \pm SEM. Results are representative of three independent experiments. Serum iron levels were measured as concentration (D) and as fold change (E) at $1.5,3,6,12$, and 24 hours after LPS $(0.1 \mu \mathrm{g} / \mathrm{g})$ or saline (Ctrl) injection in WT and $H f e^{-1-}$ mice fed a standard diet and in WT mice fed a standard diet supplemented with $2.5 \%$ carbonyl iron $(\mathrm{WT}+\mathrm{CI})$. Data are expressed as means \pm SEM. Results are representative of two similar experiments. ${ }^{*} P<0.05$, ${ }^{* *} P<0.001$ versus saline-treated mice $(\mathrm{Ctrl})$ (A-D); ${ }^{\dagger} P<0.05$ WT versus WT + CI or $H f e^{-1-}$ mice $(\mathbf{D}) . n=4$ mice per group $(\mathbf{A}-\mathbf{C}) ; n=6$ mice per group $(\mathbf{D}$ and $\mathbf{E})$.

Next, we sought to evaluate the importance of Lcn2 in the acute hypoferremic response triggered by LPS by using Lcn2-deficient mice. Serum iron levels in $\mathrm{Lcn}^{-1-}$ mice were indistinguishable from those of WT mice treated with LPS at each time point examined (Figure 7C). Thus, Lcn2 is not involved in the acute hypoferremic response elicited by LPS.

Taken together, these results show that Lcn2 is regulated by a pathway largely dependent on MyD88 signaling. In the absence of bacterial siderophores, however, Lcn2 does not participate in iron sequestration during the acute hypoferremic response elicited by LPS.

\section{Discussion}

The present study was undertaken to clarify the role played by the TLR signal adaptor molecules MyD88 and TRIF in regulating the coordinated expression of genes involved in iron metabolism during the acute hypoferremic response. Here, we show that signaling through TLRs leads to acute hypoferremia and that mice with targeted disruption of MyD88 were unable to maintain this hypoferremic response. Furthermore, MyD88 ${ }^{-1-}$ mice manifested impairment in appropriately regulating the expression of hepcidin and Lcn2, as well as in the ability to store iron in the spleen during hypoferremia.

\section{Iron Uptake and HFE Protein-TfR1 Interaction}

Here we show for the first time that Hfe expression in the spleen is down-regulated during the acute hypoferremic response in vivo. Earlier studies have shown that HFE protein and transferrin compete for the same binding sites on TfR $1,{ }^{33,34}$ and that the expression of Hfe in macrophages and monocytes inhibits iron uptake, an effect that depends on the ability of HFE protein to bind to TfR1. ${ }^{33}$ Although it is difficult to extrapolate these previous observations to in vivo systemic effects during inflammation, reduction of HFE protein levels during hypoferremia may allow for more iron-bound transferrin to associate with TfR1, thereby enhancing the iron uptake capability of the cells. Thus, our results may suggest a novel mechanism, HFE protein suppression, by which macrophages may enhance transferrin-mediated iron uptake during the hypoferremic response. The proposed role for HFE protein in regulating in vivo iron uptake is in line with recent studies showing that $\mathrm{Hfe}$ expression impairs iron uptake in erythroid cells. ${ }^{35}$ Further investigations are needed to understand whether the expression levels of Hfe can affect iron uptake via TfR1 in macrophages in response to inflammation.

TfR1, on the other hand, has been shown to be up-regulated through a pathway dependent on NF- $\mathrm{B}$ induction in the early phases of hypoferremia ${ }^{6}$ and down-regulated at later stages by proinflammatory cytokines. ${ }^{5,36}$ HFE protein down-regulation was also observed in the MyD88-deficient mice (which have impaired NF- $\kappa \mathrm{B}$ activation and induction of NF- $\kappa \mathrm{B}-$ dependent proinflammatory cytokines ${ }^{37}$ ); this finding suggests that HFE protein suppression in response to inflammation is MyD88-independent. We also found that HFE protein down-regulation is TRIF-independent, because Trif ${ }^{L p s 2 / L p s 2}$ mice treated with poly(I:C) also down-regulate HFE protein expression in the spleen. Ultimately, the coordinated up-regulation of $\operatorname{TfR} 1^{6}$ and 
A

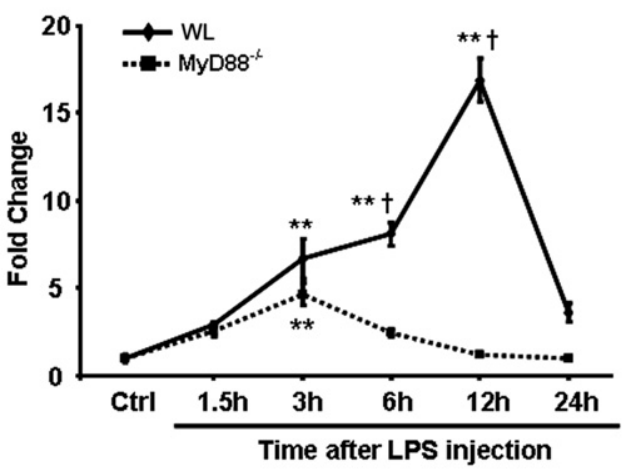

B

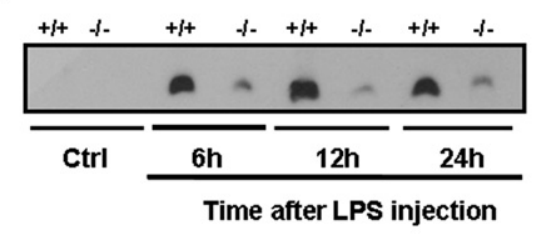

$\mathrm{C}$

Serum iron

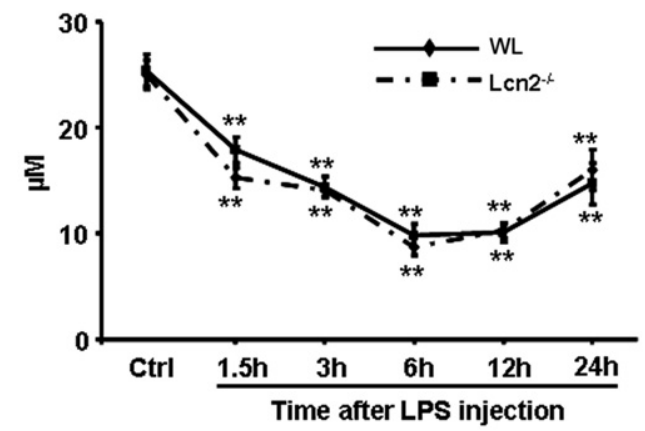

Figure 7. The role of Lcn 2 in the hypoferremic response. Lcn 2 mRNA in the spleen (A) and Lcn 2 protein levels in the serum (B) of WT $(+/+)$ and $M y D 88^{-/-}(-/-)$mice after LPS treatment. Data are expressed as means \pm SEM. Results are representative of three independent experiments. C: Serum iron levels in WT and $\mathrm{Lcn} 2^{-/-}$mice after LPS treatment. Data are expressed as means \pm SEM. ${ }^{*} P<0.05,{ }^{* *} P<0.001$ versus saline-treated mice $(\mathrm{Ctrl})$ ${ }^{\dagger} P<0.05$ WT versus $M y D 88^{-1-}$ mice. $n=4$ mice/group (A and $\left.\mathbf{B}\right) ; n=6$ mice per group $(\mathbf{C})$.

down-regulation of HFE protein may contribute to the early development of hypoferremia by increasing transferrin-mediated iron uptake from the blood.

In the present study, circulating iron dropped significantly from basal levels in $\mathrm{Hfe}^{-/-}$mice treated with LPS. However, at each time point examined, $\mathrm{Hfe}^{-\prime-}$ mice exhibited markedly higher serum iron levels, compared with WT mice. A similar outcome could be reproduced when WT mice were fed an iron-enriched diet, indicating that the reduction in circulating iron during the hypoferremic response is limited by the initial basal iron levels (rather than by the presence or absence of HFE protein). These results may help explain inconsistent findings among previous studies investigating the role of HFE protein during acute hypoferremia, ${ }^{14,38-40}$ because the severity of iron accumulation in $\mathrm{Hfe}^{-/-}$mice has been shown to differ considerably with genetic background ${ }^{14,41}$ and may additionally be affected by the standard commercial diet used. ${ }^{27}$ Furthermore, our results suggest that only a limited amount of iron can be removed from the circulation during the immediate acute hypoferremic response.

\section{Iron Storage and Sequestration}

The inability to maintain the hypoferremic response in $M y D 88^{-1-}$ mice may be associated with failure to appropriately store iron in macrophages, judging from the differences observed in iron accumulation in the spleen, a response that we found to be dependent on MyD88 signaling.

Lcn2, an important protein involved in iron sequestration, is rapidly induced on TLR activation by a pathway that we show here to be dependent on MyD88 signaling. Although iron sequestration by Lcn2 has been shown to play an important role in limiting bacterial growth during the innate immune response, ${ }^{18}$ here we show that the hypoferremic response triggered by LPS is very similar in Lcn2-deficient and WT mice. This result suggests that Lcn2 action during infection is directly related to its ability to bind ironloaded bacterial siderophores and that, in contrast, binding of Lcn2 to endogenous siderophores ${ }^{42,43}$ does not contribute to iron removal from the circulation. The importance of sequestering iron-siderophore complexes to limit iron availability to invading pathogens during the initial phase of the innate immunity response is highlighted by the finding that mice deficient in Lcn2 show substantially accelerated lethality when infected with E. coli. ${ }^{18}$

\section{Iron Release and the Ferroportin-Hepcidin Axis}

In contrast to WT mice, both MyD88- and TRIF-deficient mice were unable to up-regulate hepcidin expression on stimulation with LPS, but still retained the ability to induce hepcidin when injected with recombinant IL-6. Thus, the lack of hepcidin induction in the mutant mice seems to be attributable to the severe impairment in cytokine production (notably IL-6 in MyD88-deficient mice), as shown here and in previous studies. $^{37}$ Similarly, previous report indicates that TRIF-deficient mice exhibit impaired inflammatory cytokine production after LPS stimulation, and it has therefore been proposed that cooperation between the MyD88-dependent and MyD88-independent (TRIF-dependent) signaling pathways is required for TLR4-mediated inflammatory cytokine production. ${ }^{44}$

Although poly(l:C) treatment did not induce hepcidin expression in the liver or spleen in WT mice, splenic hepcidin has been shown to be induced in rainbow trout. ${ }^{45}$ Despite the lack of hepcidin induction, TRIFdeficient mice developed acute hypoferremia in response to LPS and to poly(I:C). These findings further support previous studies suggesting that hepcidin-independent mechanisms likely contribute to the development of acute hypoferremia induced by TLR agonists. ${ }^{14}$ 
In contrast to hepcidin regulation, ferroportin suppression during the acute hypoferremic response occurs largely independent of MyD88 and TRIF signaling. In macrophages, ferroportin expression is regulated at several levels, including transcriptional regulation, ${ }^{9,46-48}$ translational regulation, ${ }^{8,13,49}$ and posttranslational regulation by hepcidin. ${ }^{13}$ Earlier studies have shown that ferroportin expression in macrophages is regulated at the level of mRNA stability during inflammation. This has been reported to occur by a mechanism involving iron regulatory protein 1 (IRP1) binding to the iron-responsive element (IRE) ${ }^{30,50}$ found at the $5^{\prime}$ site of the ferroportin transcript. ${ }^{9}$ The IRP1/IRE translation block of ferroportin was found to be mediated by nitric oxide, ${ }^{50}$ an important physiological regulator produced by macrophages during inflammation. However, ferritin genes, which also have an IRE Iocated at the $5^{\prime}$ site, are regulated in the opposite direction by nitric oxide; that is, ferritin synthesis substantially increases and leads to increased sequestration of intracellular iron in ferritin. ${ }^{51}$ Thus, it is not clear how the IRP1/IRE mechanism may contribute to ferroportin mRNA repression during the initial phase of the inflammatory response. Nevertheless, once intracellular iron levels rise, deactivation of IRPs by iron would be expected to promote translation of ferroportin, and it is at this later phase of the inflammatory response that hepcidin may be envisioned to play a critical role in maintaining ferroportin repression. ${ }^{13}$

Ultimately, as with HFE protein, the ability to rapidly down-regulate ferroportin mRNA levels, which is retained in both $M y D 88^{-/-}$and Trif ${ }^{L p s 2 / L p s 2}$ mice, may contribute to the initial drop in serum iron levels seen in these mice.

\section{MyD88-Dependent and -Independent Regulation of Iron Homeostasis during Inflammation}

The present results suggest that the acute hypoferremic response may be regulated in at least two phases. The immediate phase allows for a rapid decrease in circulating iron and, because it is also observed in MyD88 ${ }^{-1-}$ mice, it does not depend on NF- $\kappa \mathrm{B}$ activation (MyD88- and TRIF-independent) and subsequent proinflammatory cytokine production. Iron removal from the circulation during this early phase may be mediated by the coordinated control of augmented cellular iron uptake via TfR1 due to HFE protein downregulation, and limited iron efflux through direct suppression of ferroportin expression. Indeed, suppression of HFE protein and ferroportin expression was also observed in MyD88 ${ }^{-1-}$ mice; however, because hypoferremia is not maintained in $M y D 88^{-1-}$ mice compared with WT mice, these reductions are not sufficient to sustain the hypoferremic response.

The second phase is largely MyD88- and TRIF-dependent and requires NF- $\kappa$ B activation. Binding of LPS to TLR4 initially transmits signals for the early-phase activation of NF- $\kappa \mathrm{B}$ by recruiting MyD88 (MyD88-de- pendent pathway). The TLR4-LPS complex is then internalized and retained in the endosome, where it triggers signal transduction by recruiting TRIF, which leads to activation of late-phase NF- $\kappa$ B and IRF3 for induction of type I interferon (TRIF-dependent pathway). ${ }^{2}$ Both early- and late-phase activation of NF- $\kappa \mathrm{B}$ are required for the induction of inflammatory cytokines (eg, IL-6 and TNF- $\alpha$ ), which in turn activate the expression of genes involved in the regulation of iron export (hepcidin) and sequestration (Lcn2). Binding of poly(I:C) to TLR3 activates the TRIF-dependent pathway to induce type I interferon and proinflammatory cytokines.

Additional removal of circulating siderophoreloaded iron from invading pathogens may ensue by compatible binding of bacterial siderophores to Lcn2, and further repression of ferroportin protein can be achieved through hepcidin induction. Progressive accumulation of iron within macrophages becomes detectable by 12 hours after LPS injection, as measured in spleen samples using atomic absorption spectroscopy. When sustained over long periods of time, this response may lead to the development of functional iron deficiency, with consequent limited erythropoiesis (as seen in anemia of chronic disease).

In summary, the present study provides evidence that it may be possible to interfere with the progression of hypoferremia by suppressing TLR-induced inflammatory pathways. We propose that targeting specific TLR signaling pathways by affecting the function of adaptor molecules may provide new and complementary strategies to counteract iron sequestration within macrophages triggered by immune system activation.

\section{Acknowledgments}

We thank Dr. Peter P. Liu (University of Toronto, Canada) for help with the MyD88 ${ }^{-1-}$ mouse strain and Marco Constante (Center for Genomic Regulation, Barcelona, Spain) for critically reviewing the manuscript.

\section{References}

1. Medzhitov R: Origin and physiological roles of inflammation. Nature 2008, 454:428-435

2. Kumar $\mathrm{H}$, Kawai $\mathrm{T}$, Akira $\mathrm{S}$ : Pathogen recognition by the innate immune system. Int Rev Immunol 2011, 30:16-34

3. Weiss G: Iron metabolism in the anemia of chronic disease. Biochim Biophys Acta 2009, 1790:682-693

4. Ganz T, Nemeth E: Iron sequestration and anemia of inflammation. Semin Hematol 2009, 46:387-393

5. Byrd TF, Horwitz MA: Regulation of transferrin receptor expression and ferritin content in human mononuclear phagocytes. Coordinate upregulation by iron transferrin and downregulation by interferon gamma. J Clin Invest 1993, 91:969-976

6. Tacchini L, Gammella E, De Ponti C, Recalcati S, Cairo G: Role of HIF-1 and NF-kappaB transcription factors in the modulation of transferrin receptor by inflammatory and anti-inflammatory signals. J Biol Chem 2008, 283:20674-20686

7. Donovan A, Brownlie A, Zhou Y, Shepard J, Pratt SJ, Moynihan J, Paw BH, Drejer A, Barut B, Zapata A, Law TC, Brugnara C, Lux SE, Pinkus GS, Pinkus JL, Kingsley PD, Palis J, Fleming MD, Andrews NC, Zon 
LI: Positional cloning of zebrafish ferroportin1 identifies a conserved vertebrate iron exporter. Nature 2000, 403:776-781

8. Abboud S, Haile DJ: A novel mammalian iron-regulated protein involved in intracellular iron metabolism. J Biol Chem 2000, 275:1990619912

9. McKie AT, Marciani P, Rolfs A, Brennan K, Wehr K, Barrow D, Miret S, Bomford A, Peters TJ, Farzaneh F, Hediger MA, Hentze MW, Simpson $\mathrm{RJ}$ : A novel duodenal iron-regulated transporter, IREG1, implicated in the basolateral transfer of iron to the circulation. Mol Cell 2000, 5:299-309

10. Torti SV, Kwak EL, Miller SC, Miller LL, Ringold GM, Myambo KB, Young AP, Torti FM: The molecular cloning and characterization of murine ferritin heavy chain, a tumor necrosis factor-inducible gene. J Biol Chem 1988, 263:12638-12644

11. Park $\mathrm{CH}$, Valore EV, Waring AJ, Ganz T: Hepcidin, a urinary antimicrobial peptide synthesized in the liver. J Biol Chem 2001, 276:78067810

12. Pigeon C, Ilyin G, Courselaud B, Leroyer P, Turlin B, Brissot P, Loreal $\mathrm{O}$ : A new mouse liver-specific gene, encoding a protein homologous to human antimicrobial peptide hepcidin, is overexpressed during iron overload. J Biol Chem 2001, 276:7811-7819

13. Nemeth E, Tuttle MS, Powelson J, Vaughn MB, Donovan A, Ward DM, Ganz T, Kaplan J: Hepcidin regulates cellular iron efflux by binding to ferroportin and inducing its internalization. Science 2004, 306:2090-2093

14. Constante M, Jiang W, Wang D, Raymond VA, Bilodeau M, Santos MM: Distinct requirements for Hfe in basal and induced hepcidin levels in iron overload and inflammation. Am J Physiol Gastrointest Liver Physiol 2006, 291:G229-G237

15. Constante M, Wang D, Raymond VA, Bilodeau M, Santos MM: Repression of repulsive guidance molecule $\mathrm{C}$ during inflammation is independent of $\mathrm{Hfe}$ and involves tumor necrosis factor-alpha. Am J Pathol 2007, 170:497-504

16. Feder JN, Gnirke A, Thomas W, Tsuchihashi Z, Ruddy DA, Basava A, Dormishian F, Domingo R Jr, Ellis MC, Fullan A, Hinton LM, Jones NL, Kimmel BE, Kronmal GS, Lauer P, Lee VK, Loeb DB, Mapa FA, McClelland E, Meyer NC, Mintier GA, Moeller N, Moore T, Morikang E, Wolff RK: A novel MHC class I-like gene is mutated in patients with hereditary haemochromatosis. Nat Genet 1996, 13:399-408

17. Pietrangelo A: Molecular insights into the pathogenesis of hereditary haemochromatosis. Gut 2006, 55:564-568

18. Flo TH, Smith KD, Sato S, Rodriguez DJ, Holmes MA, Strong RK, Akira S, Aderem A: Lipocalin 2 mediates an innate immune response to bacterial infection by sequestrating iron. Nature 2004, 432:917-921

19. Nairz M, Theurl I, Schroll A, Theurl M, Fritsche G, Lindner E, Seifert M, Crouch M-LV, Hantke K, Akira S, Fang FC, Weiss G: Absence of functional Hfe protects mice from invasive Salmonella enterica serovar Typhimurium infection via induction of lipocalin-2. Blood 2009, 114:3642-3651

20. Adachi O, Kawai T, Takeda K, Matsumoto M, Tsutsui H, Sakagami M, Nakanishi K, Akira S: Targeted disruption of the MyD88 gene results in loss of IL-1- and IL-18-mediated function. Immunity 1998, 9:143-150

21. Levy JE, Montross LK, Cohen DE, Fleming MD, Andrews NC: The C282Y mutation causing hereditary hemochromatosis does not produce a null allele. Blood 1999, 94:9-11

22. Miranda CJ, Makui H, Andrews NC, Santos MM: Contributions of beta2-microglobulin-dependent molecules and lymphocytes to iron regulation: insights from HfeRag1(-/-) and beta2mRag1(-/-) double knock-out mice. Blood 2004, 103:2847-2849

23. Gkouvatsos K, Wagner J, Papanikolaou G, Sebastiani G, Pantopoulos $\mathrm{K}$ : Conditional disruption of mouse $\mathrm{Hfe} 2$ gene: maintenance of systemic iron homeostasis requires hepatic but not skeletal muscle hemojuvelin. Hepatology 54:1800-1807

24. Coppola G, Choi S-H, Santos MM, Miranda CJ, Tentler D, Wexler EM, Pandolfo M, Geschwind DH: Gene expression profiling in frataxin deficient mice: microarray evidence for significant expression changes without detectable neurodegeneration. Neurobiol Dis 2006 22:302-311

25. Makui H, Soares RJ, Jiang W, Constante M, Santos MM: Contribution of Hfe expression in macrophages to the regulation of hepatic hepcidin levels and iron loading. Blood 2005, 106:2189-2195

26. Nicolas G, Chauvet C, Viatte L, Danan JL, Bigard X, Devaux I, Beaumont $C$, Kahn A, Vaulont S: The gene encoding the iron regu- latory peptide hepcidin is regulated by anemia, hypoxia, and inflammation. J Clin Invest 2002, 110:1037-1044

27. Nemeth E, Rivera S, Gabayan V, Keller C, Taudorf S, Pedersen BK, Ganz T: IL-6 mediates hypoferremia of inflammation by inducing the synthesis of the iron regulatory hormone hepcidin. J Clin Invest 2004, 113:1271-1276

28. Donovan A, Lima CA, Pinkus JL, Pinkus GS, Zon LI, Robine S, Andrews NC: The iron exporter ferroportin/Slc40a1 is essential for iron homeostasis. Cell Metab 2005, 1:191-200

29. Peyssonnaux C, Zinkernagel AS, Datta V, Lauth X, Johnson RS, Nizet V: TLR4-dependent hepcidin expression by myeloid cells in response to bacterial pathogens. Blood 2006, 107:3727-3732

30. Yang F, Liu XB, Quinones M, Melby PC, Ghio A, Haile DJ: Regulation of reticuloendothelial iron transporter MTP1 (Slc11a3) by inflammation. J Biol Chem 2002, 277:39786-39791

31. Theurl I, Aigner E, Theurl M, Nairz M, Seifert M, Schroll A, Sonnweber T, Eberwein L, Witcher DR, Murphy AT, Wroblewski VJ, Wurz E, Datz C, Weiss G: Regulation of iron homeostasis in anemia of chronic disease and iron deficiency anemia: diagnostic and therapeutic implications. Blood 2009, 113:5277-5286

32. Roy CN, Penny DM, Feder JN, Enns CA: The hereditary hemochromatosis protein, HFE, specifically regulates transferrin-mediated iron uptake in HeLa cells. J Biol Chem 1999, 274:9022-9028

33. Drakesmith H, Sweetland E, Schimanski L, Edwards J, Cowley D, Ashraf M, Bastin J, Townsend ARM: The hemochromatosis protein HFE inhibits iron export from macrophages. Proc Natl Acad Sci USA 2002, 99:15602-15607

34. Giannetti AM, Bjorkman PJ: HFE and transferrin directly compete for transferrin receptor in solution and at the cell surface. J Biol Chem 2004, 279:25866-25875

35. Ramos P, Guy E, Chen N, Proenca CC, Gardenghi S, Casu C, Follenzi A, Van Rooijen N, Grady RW, de Sousa M, Rivella S: Enhanced erythropoiesis in Hfe-KO mice indicates a role for Hfe in the modulation of erythroid iron homeostasis. Blood 2011, 117:1379-1389

36. Kim S, Ponka P: Effects of interferon-gamma and lipopolysaccharide on macrophage iron metabolism are mediated by nitric oxide-induced degradation of iron regulatory protein 2. J Biol Chem 2000, 275:6220-6226

37. Kawai T, Adachi O, Ogawa T, Takeda K, Akira S: Unresponsiveness of MyD88-deficient mice to endotoxin. Immunity 1999, 11:115-122

38. Frazer DM, Wilkins SJ, Millard KN, McKie AT, Vulpe CD, Anderson GJ: Increased hepcidin expression and hypoferraemia associated with an acute phase response are not affected by inactivation of HFE. Br J Haematol 2004, 126:434-436

39. Lee P, Peng H, Gelbart T, Beutler E: The IL-6- and lipopolysaccharide-induced transcription of hepcidin in HFE-, transferrin receptor 2-, and beta 2-microglobulin-deficient hepatocytes. Proc Natl Acad Sci USA 2004, 101:9263-9265

40. Roy CN, Custodio AO, de Graaf J, Schneider S, Akpan I, Montross LK, Sanchez M, Gaudino A, Hentze MW, Andrews NC, Muckenthaler $\mathrm{MU}$ : An Hfe-dependent pathway mediates hyposideremia in response to lipopolysaccharide-induced inflammation in mice. Nat Genet 2004, 36:481-485

41. Fleming RE, Holden CC, Tomatsu S, Waheed A, Brunt EM, Britton RS, Bacon BR, Roopenian DC, Sly WS: Mouse strain differences determine severity of iron accumulation in Hfe knockout model of hereditary hemochromatosis. Proc Natl Acad Sci USA 2001, 98 2707-2711

42. Devireddy LR, Hart DO, Goetz DH, Green MR: A mammalian siderophore synthesized by an enzyme with a bacterial homolog involved in enterobactin production. Cell 2010, 141:1006-1017

43. Bao G, Clifton M, Hoette TM, Mori K, Deng S-X, Qiu A, Viltard M, Williams D, Paragas N, Leete T, Kulkarni R, Li X, Lee B, Kalandadze A, Ratner AJ, Pizarro JC, Schmidt-Ott KM, Landry DW, Raymond KN, Strong RK, Barasch J: Iron traffics in circulation bound to a siderocalin (Ngal)-catechol complex. Nat Chem Biol 6:602-609

44. Yamamoto M, Sato S, Hemmi H, Hoshino K, Kaisho T, Sanjo $H$, Takeuchi O, Sugiyama M, Okabe M, Takeda K, Akira S: Role of adaptor TRIF in the MyD88-independent Toll-like receptor signaling pathway. Science 2003, 301:640-643

45. Chiou PP, Lin CM, Bols NC, Chen TT: Characterization of virus/ double-stranded RNA-dependent induction of antimicrobial pep- 
tide hepcidin in trout macrophages. Dev Comp Immunol 2007, 31:1297-1309

46. Knutson MD, Vafa MR, Haile DJ, Wessling-Resnick M: Iron loading and erythrophagocytosis increase ferroportin 1 (FPN1) expression in J774 macrophages. Blood 2003, 102:4191-4197

47. Harada N, Kanayama M, Maruyama A, Yoshida A, Tazumi K, Hosoya T, Mimura J, Toki T, Maher JM, Yamamoto M, Itoh K: Nrf2 regulates ferroportin 1-mediated iron efflux and counteracts lipopolysaccharide-induced ferroportin $1 \mathrm{mRNA}$ suppression in macrophages. Arch Biochem Biophys 508:101-109

48. Marro S, Chiabrando D, Messana E, Stolte J, Turco E, Tolosano E, Muckenthaler MU: Heme controls ferroportin1 (FPN1) transcription involving Bach1, Nrf2 and a MARE/ARE sequence motif at position -7007 of the FPN1 promoter. Haematologica 95:1261-1268

49. Lymboussaki A, Pignatti E, Montosi G, Garuti C, Haile DJ, Pietrangelo $A$ : The role of the iron responsive element in the control of ferroportin1/IREG1/MTP1 gene expression. J Hepatol 2003, 39: $710-715$

50. Liu XB, Hill P, Haile DJ: Role of the ferroportin iron-responsive element in iron and nitric oxide dependent gene regulation. Blood Cells Mol Dis 2002, 29:315-326

51. Kim S, Ponka P: Nitrogen monoxide-mediated control of ferritin synthesis: implications for macrophage iron homeostasis. Proc Natl Acad Sci USA 2002, 99:12214-12219 